\title{
Transcatheter arterial chemoembolization and radiofrequency ablation combined with immunotherapy for hepatocellular carcinoma patients
}

\author{
Liang Wang, Zhong-Qiang Yu, Ke-Lin Yao, Jing-Song Hu, Shao Gao, Lv-Cong Zhu, Rui-Ming Xia
}

Department of Radiology, the Affiliated Hospital of Shaoxing University (Shaoxing Municipal Hospital), Shaoxing, Zhejiang Province, China

Submitted: 4 October 2021; Accepted: 6 February 2022

Online publication: 18 February 2022

Arch Med Sci

DOI: https://doi.org/10.5114/aoms/146438

Copyright $\odot 2022$ Termedia \& Banach

\section{Abstract}

Successive trials using immune response checkpoint blockade, the systemic therapy for progressive hepatocellular carcinoma (HCC), have shifted dramatically towards targeting the tumor microenvironment (TME). Locoregional therapies (LRTs) such as radiofrequency ablation (RFA)/rhizotomy, trans-arterial chemoembolization (TACE) and surgical resection in the initial stages are critical. LRTs perform the crucial task of modulating the immune system surrounding the tumor, which is of great importance in the prevailing era of developing immuno-oncology treatments and their direct effect on tumor cells. Significant efforts are needed in exploring the mechanisms of the latest combinational approach to boost the clinical advantage with tolerable side effects. The present review highlights the tumor microenvironment of HCC followed by current immunotherapeutic approaches for HCC. Next, we discuss the concealed mechanisms of LRTs given in combination with immunotherapy and their utilization in clinical practice.

Key words: hepatocellular carcinoma, transcatheter arterial chemoembolization, radiofrequency ablation, immunotherapy.

\section{Introduction}

One of the most familiar liver diseases is hepatocellular carcinoma (HCC) and it is the third leading cause of death among all types of cancers $[1,2]$. Treatment options are available from locoregional therapies (LRTs) for a localized tumor to systemic treatments for metastatic HCC or multifocal carcinoma, depending on the nature of the disease. Three LRTs, i.e., trans-arterial chemoembolization (TACE), radiofrequency ablation (RFA) and cryoablation, are recommended in patients whose immune system rejects liver transplant or surgical resection. However, most patients ultimately develop a refractory disease that needs systemic treatment [3]. Currently, the only FDA approved systemic drug is sorafenib. Regorafenib [4], ramucirumab [5], cabozantinib [6], and lenvatinib are examples of targeted treatment that have shown efficacy in first-line and second-line treatment in HCC and are certified by the FDA. On the other hand, programmed cell death protein-1 (PD-1) inhibitors such as pembrolizumab and nivolumab have achieved approval by the FDA only in the second-line environment [7, 8]. Given the proven efficacy of each treatment option in HCC, the debate is whether it is reasonable to continue

\author{
Corresponding author: \\ Dr. Rui-Ming Xia \\ the Affiliated Hospital \\ of Shaoxing University \\ (Shaoxing Municipal Hospital) \\ 999 Zhongxing South Road \\ Shaoxing 312000 \\ Zhejiang Province, China \\ Phone: +86-0575-88293701 \\ Fax: +86-0575-88293113 \\ E-mail: xiaruim@163.com
}


the immunotherapy and TACE combination, or the moderate effect of immunotherapy can be transferred to the adjuvant setting post-TACE in the advanced setting. The current review highlights the tumor microenvironment of HCC followed by current immunotherapeutic approaches for HCC. Next, we discuss the concealed mechanisms of LRTs given in combination with immunotherapy and their utilization in clinical practice.

\section{Immune landscape of HCC}

The liver is often inflamed as an outcome of chronic hepatitis infection or long-term liver injury [9]. However, mild inflammation acts as a barrier against pathogens and helps to heal tissue injury, whereas persistent liver inflammation may disrupt the microenvironment and aggravate carcinogenesis [10-12]. The liver may generate inflammatory mediators such as chemokines and cytokines, which accelerate angiogenesis, anti-apoptotic cascades, and immune evasion as well as attracting immunocytes capable of creating a niche microenvironment that promotes tumor development [13-15]. Tumor-linked chemokines and cytokines can engage and polarize immune molecules into a tumor supporting phenotype, thereby promoting tumor development. Tumor-associated macrophages are polarized into M2 macrophages by IL-4, IL-10, IL-13, and TGF-b, which exist in the TME and initiate development of tumor by recruiting CD4+FoxP3+ regulatory T-cells (Tregs) (Figure 1) and stimulating angiogenesis, constituting one such immune subset $[16,17]$. TAM accumulation in the tumor region is linked to a weak diagnosis in various cancers, including HCC $[16,18]$.

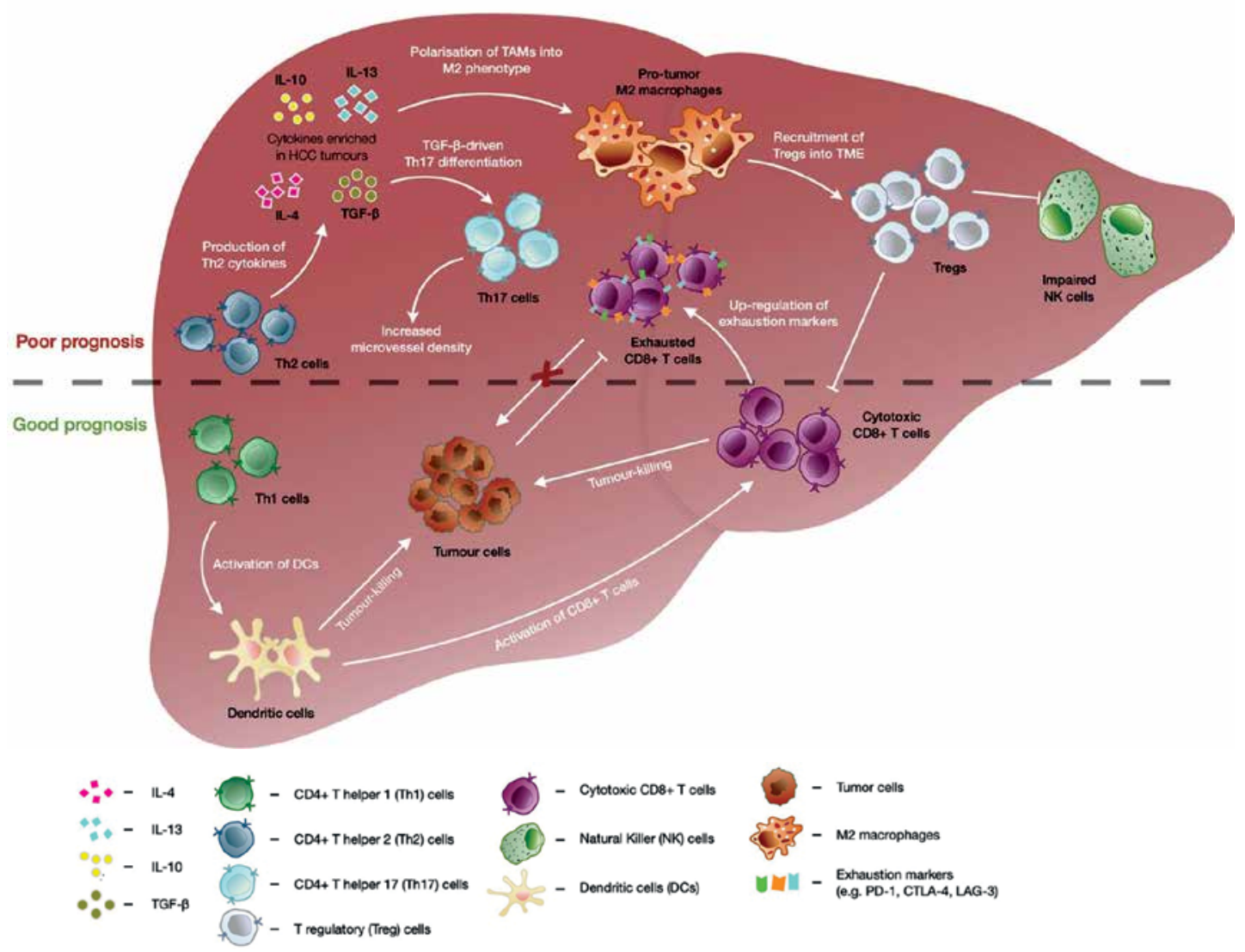

Figure 1. The immune system episodes contribute to a favorable or unfavorable mechanism in HCC. CD4+ Thelper 2 (Th2) cell enrichment is linked to a weak overall survival rate in patients with HCC. TGF-b and Th2- generates cytokines such as IL-4, IL-10, and IL-13, that promote tumor-linked macrophages (TAMs) to polarize into M2 macrophage phenotype as an immunosuppressive agent that attracts T regulatory cells (Tregs). Particularly, Tregs improve the immunosuppressive tumor surrounding environment by blocking the pathway of natural killer cells and CD8+ T memory/effector cells that possess tumor-killing capability that weakens disease prognosis. TGF-b also assisted in CD4+ T helper 17 (Th17) cell differentiation and increased Th17 cell prevalence, linked to enhanced microvascular aggression and decreased DFS and OS in patients with HCC. Since CD4+ T helper 1 cells (Th1) can enhance CD8+ T cell excitation with the help of dendritic cells (DC) and stimulate DC-mediated tumor suppression, their presence was linked to better outcomes in HCC. Despite their ability to destroy tumor cells and their connection to a better prognosis in HCC, upregulation of fatigue biomarkers is shown by CD8+ T cells (e.g., LAG-3, PD-1 and CTLA-4), inhibiting their killing potential in chronic conditions and leading to tumor progression. Reproduced with permission from Lee et al. [39] 
Tregs, which control or dampen the cytotoxic potential and activate CD8-positive T cells, act as an integral component in tumorigenesis [19, 20]. Earlier, we stated that Tregs from HBV-positive hepatocellular carcinoma showed increased expression of PD-1 and were more effective at suppressing CD8-positive $T$ cells. Treg production by higher intra-tumoral interleukin-17 has been regularly identified in HCC patients, linked to a worse prognosis and shorter survival $[12,18,19$, 21, 22]. TGF-b-rich HCC TME also promotes Th17 separation; a CD4+ $\mathrm{T}$ subgroup also produces IL-17 [23]. In HCC patients, high intra-tumoral Th17 frequencies were linked to microvascular invasion and a weak overall survival rate (OS) along with disease-free survival (DFS) [24].

CD4+ T helper 1 cells (Th1) can produce IFN-g, and on the other hand can activate dendritic cells, resulting in improved excitation and advancement of CD8+ T cells $[25,26]$. Th1 helper cells also activate dendritic cell-mediated tumor suppression via IFN- $\gamma$-dependent pathways, fueling anti-tumor immune responses downstream (Figure 1) [27]. Patients with HCC are treated with transarterial chemoembolization (TACE); an improvement in Th1 response was linked to better outcomes [28]. Though Th1 has demonstrated anti-tumor activity, compared to normal healthy livers, type 2 CD4+ $\mathrm{T}$ helper cells (Th2) cells were augmented in HCC tissues and are conversely related with overall survival of HCC patients [29]. Th2-type cytokines such as interleukin-10 (IL-10), interleukin-4 (IL-4) and interleukin-13 (IL-13) will make M2 macrophages less cytotoxic, reducing CD8+ T cell-initiated cytotoxic activity (Figure 1) [30, 31]. Moreover, increased Th2 cytokine output has been associated with an enhanced risk of HCV- HCC $[32,33]$.

Immune molecules that are cytotoxic, such as natural killer cells (NK), CD8+ T effector memory (Tem) and CD8+ T resident memory (Trm), act as anti-tumor agents $[18,21]$. However, natural killer cells around the HCC tumor regions were found to have lower cytolytic ability and IFN-g output than NK cells from a healthy cellular environment [34]. Lee stated that the Tregs obtained from HCC patients showed a reduction in the tumor-suppressing capacity of homologous NK cells (Figure 1). Although the existence of Tem and Trm cells can be a bridge for better prediction in HCC [35], they frequently release biomarkers including lymphocyte-activation gene 3 (LAG-3), cytotoxic T-lymphocyte-associated antigen 4 (CTLA-4) and PD-1 that are adversely linked with functional ability (Figure 1) [36-38]. The detailed landscape of HCC is shown in Figure 1 [39].

\section{Immunotherapy in HCC (immune checkpoint blockers)}

PD-1 inhibitors such as nivolumab and pembrolizumab in second-line treatment have shown their recent involvement in mature and metastatic HCC after sorafenib. Pembrolizumab and nivolumab were approved by the FDA after conducting two different clinical trials, the Keynote-224 on 105 patients and Checkmate 040 on 262 patients, respectively $[40,41]$. Both the trials were Phase II stage single arm and open-label experiments on patients with metastatic HCC resistant to sorafenib. Patients with Child-Pugh class A were included in both trials. However, patients with B7 were included in Checkmate 040 regardless of hepatitis B or C. Pembrolizumab had a $17 \%$ overall objective response rate and nivolumab had a $20 \%$ overall unbiased response rate with a greater number of responding patients showing long-term responses. Without considering whether the patients had infectious hepatitis or not, the therapy was well accepted. Program cell death ligand 1 (PD-L1) expression did not appear to be related to nivolumab response. Although a phase III staged placebo-controlled trial (Keynote-240) in second-line treatment found that pembrolizumab reduced the risk of death by $20 \%$, it fell short of its statistical goals [42].

In the front-line environment, the findings of a recently published randomized phase III clinical trial (Checkmate 459) distinguishing nivolumab and sorafenib did not meet the previously stated statistical significance for overall survival (OS), i.e., $\mathrm{HR}=0.84, p=0.0419$, despite significant improvements in OS (16.4 months vs. 14.7 months), ORR (15\% vs. $7 \%$ ) and CR (4 vs. 1) with nivolumab. PD-L1 negative and positive tumors showed responses. $22 \%$ registered against nivolumab related side effects; however, $49 \%$ reported side effects against sorafenib [43]. Other checkpoint inhibitors of immune cells have shown minimal activity in progressed HCC. The PD-L1 antibody durvalumab showed a $10 \%$ overall response rate in pre-treated HCC patients [44]. Cytotoxic T lymphocyte-associated antigen 4 (CTLA-4) is a tremelimumab antibody that showed a $17 \%$ response, equivalent to PD-L1 or PD-1 inhibitors, but only in a limited population of 20 patients who rejected the options of surgery or LRTs in chronic hepatitis $C$ and progressed HCC [45]. PD-L1 negative and positive tumors showed responses. 22\% registered against nivolumab related side effects; however, $49 \%$ reported side effects against sorafenib [44]. In the HIMALAYA Phase 3 trial, identical tremelimumab and durvalumab were distinguished from sorafenib in the first-line treatment [46].

Anti-PD-1/PD-L1 or anti-CTLA-4 antibodies have been combined with alternative approaches such as anti-angiogenesis in a number of ongoing trials. In the most recent study, named IMbrave150, the combined effect of bevacizumab and atezolizumab (anti-PD-L1) showed a targeted response to vascular endothelial growth factor A (VEGF-A), an approach that has shown the dominant effect in 
improving progression-free survival (PFS) and OS in comparison to first-line treatment with sorafenib. The average effect of the bevacizumab and atezolizumab combination on OS was not achieved after 8.6 months of median follow-up as seen with sorafenib for 13.2 months ( $95 \% \mathrm{Cl}$ : 0.42-0.79; hazard ratio $(\mathrm{HR})=0.58 ; p=0.0006)$. However, with the combination the median PFS was found to be 6.8 months, whereas with sorafenib PFS was 4.5 months $(95 \% \mathrm{Cl}, \mathrm{HR}=0.59)$ There were continuous adverse episodes in the safety chart of all the drugs separately as well as in the combination regimen [47].

In conclusion, immune checkpoint inhibitors, when taken as a single agent, have shown an average response in the second-line setting against HCC followed by sorafenib, whereas the combinational effect has demonstrated that anti-angiogenesis leads to the front-line treatment setting due to its promising potential results.

\section{Local ablation technique and immune regulation}

Local ablation technique is now advised as one of the most efficient treatment options available for early-stage HCC. Microwave ablation (MWA) and radiofrequency ablation (RFA) are the two most frequently used thermal/energy-based ablation modalities. By introduction of energy and high temperature into the tumor, the combination together can trigger inflammatory signaling molecules, stimulate tumor necrosis and produce neoantigens, leading to anti-tumor effects [48, 49]. Cryoablation (Cryo), unlike heat-based thermal ablation, causes cell inflammation by freezing tumor cells [50]. Another non-thermal ablation method is irreversible electroporation (IRE). Inducing milliseconds of electrical pulses results in increased permeability of the cell membrane and ultimately cell apoptosis, as opposed to the thermal modality. Furthermore, it does little damage to the extracellular environment, preserving the tissues and structural constituents in their entirety [51]. The secretion of a cytokine known as macrophage migration inhibitory factor (MIF) that stimulates immune cells is the most significant effect of immunological IRE compared to RFA after the HCC procedure [52]. The mechanisms by which ablation can modulate immunity are as follows.

\section{Delivery of tumor-affiliated antigen}

Tumor necrosis caused by ablation can result in cross-presentation, tumor-associated antigen (TAA) release, and dendritic cell (DC) recruitment. Antigen related cellular immunity to T cells may be stimulated by inflammatory cytokines secreted from the local microenvironment [53]. TAA is a requirement for the immune response. Intracellular components, including highly immunogenic proteins such as heat shock protein 70 (HSP-70), are released when a tumor is destroyed; therefore, it stimulates antigen-presenting cells (APCs), inducing an antitumor immune reaction through the MHC-1 signaling pathway [54] A specific epitope of $\alpha$-fetoprotein (AFP) can also stimulate a mild-to-moderate $C D 8+T$ response in peripheral blood mononuclear cells (PBMCs) [55]. The imbalance of pro- and anti-inflammatory molecules in these disorders implies that treatment efforts aimed at suppressing excessive inflammation may benefit in disease recovery [56].

\section{Changing the level of cytokine expression}

Ablation/abscission elicits an intense immunological response on the edge of the local region. In fact, due to the need for liver homeostasis, tissue can initiate the self-mending process. Macrophages present at the wound site can secrete local cytokines [57]. Ablation helps to boost the expression of immunostimulating proteins such as tumor necrosis factor- $\alpha$ (TNF- $\alpha$ ), interferons (IFN), and interleukins (IL-1, IL-2), while silencing immunosuppressive proteins such as hepatocyte growth factor (HGF) and soluble IL-2 receptor. It also increases the CD4+/CD8+ expression ratio by provoking natural killer (NK) cells, CD3+ T/CD4+ T cells and lymphokine-activated killer cells (LAK) to become more active $[58,59]$.

\section{Motivating the immune mechanism of lymphocytes}

After peripheral blood ablation, a significant increase in movement of $C D 3+T / C D 4+T$ cells was observed. The ratio of CD45RO+, CD3+, CD68+ and CD56+ cells in the local tumor region also improves, which can last up to 30 days [60]. The effects of cytotoxic T lymphocytes (CTLS) on locally situated tumors and the immune responses are enhanced by aggregation of tumor-infiltrating lymphocytes (TILS) and circulating peripheral lymphocytes. Nevertheless, ablation can assist tumor growth, particularly after an incomplete ablation process. Ablation can also trigger cell proliferation in HCC by upregulating the IL-L6, VEGF, HIF-1, and PD-1 expression $[61,62]$ As a result, it is worth paying more attention to the resources to conduct expanded research on combinational ablation and immunotherapy.

\section{Radiation therapy and $\mathrm{ICls}$}

Immunoregulation necessitates the presence of RT. It causes tumor cells to release neoantigens, stimulates naive T-cell differentiation and proliferation, and improves T-cell functioning when given together with a PD-1 inhibitor [63]. Since the introduction of immunomodulators, radiation-induced 
abscopal effects have gained enough in preclinical models [64-66]. In an animal experiment, supporting anti-PD-L1 therapy. Therefore, this combination extended the mice's survival $(p=0.001)$ when compared to radiotherapy or anti-PD-L1 therapy alone [67]. Another study by Yoshimoto et al. revealed that RT, along with anti-CTLA-4, would boost anti-tumor effects of the immune system and prolong tumor development duration in a mouse model, which was found to be from 13.1 to 19.5 days [68]. Because of its accurate radiation delivery to the targeted lesions with less damage to the surrounding healthy tissue, selective internal radiotherapy (SIRT) is now a more acceptable option in HCC treatment [69]. Transcatheter arterial radioembolization (TARE) uses the hepatic artery to deliver radioactive particles to the target tumor, becoming a stable internal radioactive source. 1311-labeled iodized oil and 90Y microspheres are among the radioactive materials [70]. The immunocompetence of the TME has been confirmed to improve after $90 \mathrm{Y}$ therapy. This is due to increased TNF expression on the CD8+ T and CD4+ cell surface and an improvement in the ratio of APCs [71]. Figure 2 shows the various locoregional therapies and immunomodulation [72].

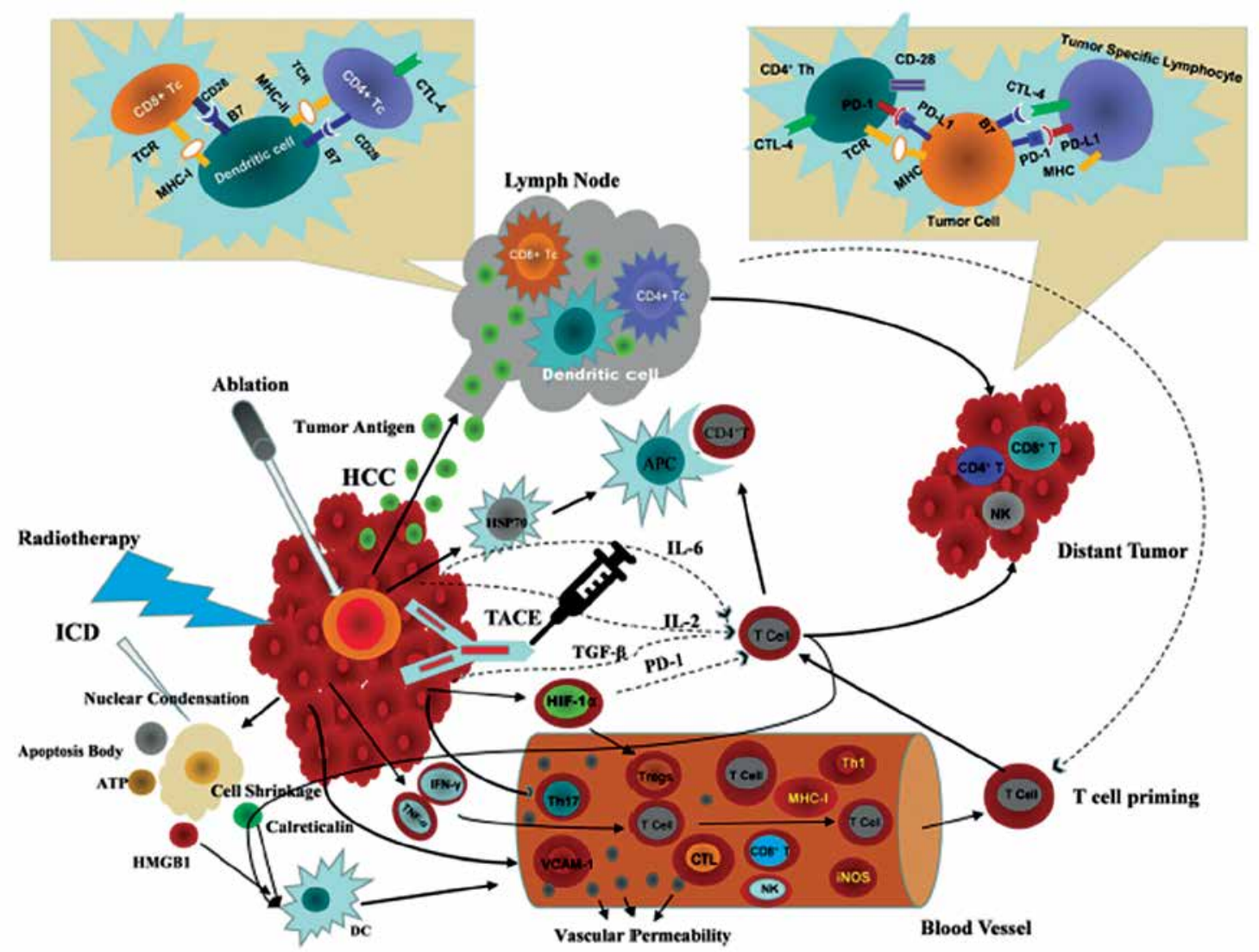

Figure 2. After peripheral blood ablation, the activity of CD3+T/CD4+ T cells increases abruptly. The population of CD3+, CD45RO+, CD56+, and CD68+ cells in the local tumor increases, which can last up to 30 days. The effects of cytotoxic T lymphocytes (CTLS) on local tumors and the immune responses are enhanced by aggregation of tumor-infiltrating lymphocytes (TILs) and circulating peripheral lymphocytes. On the other hand, ablation has the potential to initiate tumor growth, particularly after the incomplete ablation process. Hepatocellular carcinoma locoregional therapy immunomodulation mechanism. EBRT, TACE, or brachytherapy can be responsible for local tumor cell death and release immunogenic proteins such as ATP and HSP70 and TAA, which initiate APC and introduce antigens through the MHC-I pathway. Meanwhile, it attracts dendritic cells to the growing tumors and amplifies costimulatory signaling molecules, allowing tumor-specific $T$ cells to enter the blood circulation and promoting the anti-tumor effect of CTL. Treg undergoes infiltration from local tumor tissue and spreads to distant non-treated lesions, causing them to regress. Locoregional therapy alters cytokine expression and TME, which modulates tumor immunity. Simultaneously, LRT can cause immunosuppressive agents to produce (IL-6, HIF-1, TGF- $\beta$, PD-1, Tregs, and so on) that promote tumor progression. The combination of locoregional and immune therapy is thought to be promising. Tumor-associated antigens (TAA), effector T cells (Teffs), cytotoxic T lymphocyte (CTL), tumor microenvironment (TME), antigen-presenting cell (APC), regulatory T cells (Tregs), major histocompatibility complex-I (MHC-I), major histocompatibility complex II (MHC-II), programmed cell death protein 1 (PD-1), T cell receptor (TCR), cytotoxic T lymphocyte-associated protein 4 (CTLA-4), high-mobility group protein (HMGB1), vascular cell adhesion molecule-1 (VCAM-1), immunogenic cell death (ICD), transforming growth factor- $\beta$ (TGF- $\beta$ ), transcatheter arterial chemoembolization (TACE), interferon- $\gamma$ (IFN- $\gamma$ ), hypoxia-inducible factor-1 $\alpha$ (HIF-1 $\alpha$ ), interleukin-2 (IL-2), interleukin-6 (IL-6), heat shock protein 70 (HSP70). Reproduced with permission from [72] 


\section{TACE and immunomodulation}

Transcatheter arterial chemoembolization (TACE) is a popular technique for irresistible HCC with a reserved liver function that may possess immune system regulating properties. After occlusion of feeding arteries, TACE can cause local tumor necrosis and release tumor-specific antigens secured by APCs and trigger tumor-specific immune responses. TACE has also been shown to alter the cytokine spectrum, T cell activity levels, and immune cell subsets [73, 74]. It increases IFN- and IL-1 secretion while suppressing IL-10 expression. It may also increase the expression of T helper 17 cells (Th17). In patients with low Treg cell expression, TACE can shift the tumor microenvironment (TME) to Th1 dominance, resulting in a better prognosis [75]. As compared to preTACE levels, CD4+ and CD25+ Tregs in the peripheral blood decreased dramatically (7.58 to $2.65 \%$, $p=0.05)$, whereas the ratio of CD4+ to CD8+ increases significantly (30.52 \pm 4.19 vs. $1.29 \pm 0.14)$ [76]. However, TACE can also manage to accelerate histanoxia, leading to an increase in expression of PD-L1, HIF- $1 \alpha$ and aggregation of Tregs, ultimately resulting in immunological tolerance. For this reason, the disease prognosis of patients who have been subjected to TACE is largely based on the stability of tumor immune rescue and anti-tumor immunity $[75,77]$. TACE damages the bile ducts and liver (Figure 3). Hence, it is crucial to conduct curative TACE when the tumor is localized and controllable. Arachidonic acid and other polyunsaturated fatty acids in combination with immune checkpoint inhibitors and tumor infiltrating lymphocytes enhance the activity of T, NK, and macrophages, thereby assisting in tumor cell elimination and sup- pressing inflammatory side effects associated with immune checkpoint inhibitors $[78,79]$.

\section{TACE combined with ICls}

TACE begins to necrotize the tumor by blocking the way of the tumor's nourishing artery, triggering a tumor-specific immune response, and secreting neoplastic antigens. Meanwhile, it promotes anoxia, which increases the release of immunosuppressive agents (Tregs, PD-1, and MDSCs) and immune resistance [80]. As a result, tumors may become immune-tolerant after one-time TACE treatment. TACE and ICls together can improve the overall anti-tumor response. The CTLA-4 inhibitor tremelimumab and TACE have shown safety and affordability in a pilot study of HCC patients [45]. A dose of $15 \mathrm{mg} / \mathrm{kg}$ of tremelimumab for 90 days before tumor progression or extreme toxicity was given intravenously in the study. The result provides an acceptable safety profile and none of the patients needed steroids for any serious immune initiated adverse effects. After the first dose, some patients encountered a temporary but serious increase in transaminases, and it did not occur in consecutive cycles. The limited response rate was found to be $17.6 \%$, disease management was found to be $76.4 \%$, and duration of development was 6.48 months (95\% confidence interval: 3.95-9.14) [45, 81].

Tremelimumab was administered to HCC patients already receiving sorafenib in other clinical trials. Six doses of tremelimumab in 4 weeks was prescribed, followed by intravenous administrations every 3 months until the patient reached off-treatment criteria. DEB-TACE was given to pa-

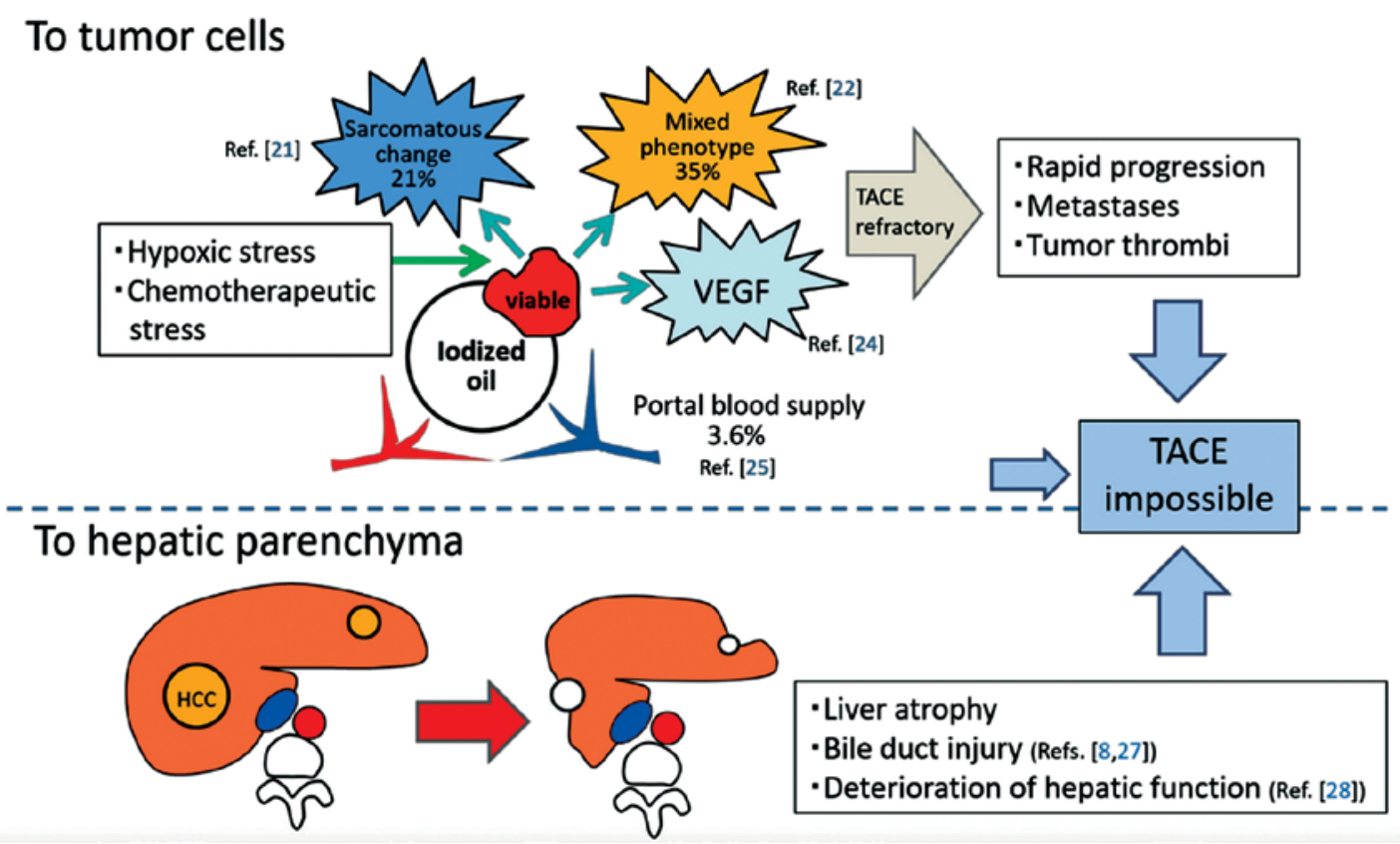

Figure 3. Showing the negative impact of TACE. Reproduced with permission from [79] 
tients on day 35 (drug-eluting bead TACE). At 2, 6, and 14 months, CT scans were performed. After 6 months the primary lesion shrank and nearly vanished, while the lesions of the non-treated intrahepatic region shrank gradually [82]. One case at our center was a 47-year-old male patient with Child-Pugh Class A of HCC who was treated with a combination of a recombinant humanized monoclonal anti-VEGF antibody (IBI305) and a fully humanized Ig 4 anti-PD-1 monoclonal antibody (sintilimab Tyvyt); both are certified for a clinical trial by the National Medical Products Administration (NMPA) of China (NCT03794440). The patient had been treated five times with TACE in the 8 months following his diagnosis. The patient agreed to take combinational therapy of IBI305 and sintilimab with TACE after the primary lesions shrank partly and new lesions appeared. Zhang et al. reported that the new combination of locoregional ablation and allogeneic cell adoption was safe and showed promising clinical effectiveness against HCC and ICC [83].

Intravenous administration of $\mathrm{IBI} 305$ and sintilimab was given with a three-week gap at a dose of $900 \mathrm{mg}$ and $200 \mathrm{mg}$ each, for a total of five doses. Tracking the progression of lesions was performed every 6 weeks and the patient was subjected to contrast-enhanced CT scans (one scan performed after every 2 doses). The total area of different lesions was reduced significantly when observed in CT images, involving partial reduction that was attained steadily right before the death endpoint. The case showed a beneficial primary outcome when IBI305 and sintilimab were given in combination with TACE to cure HCC. Another experiment conducted with nivolumab in patients with progressed HCC (NCT 03143270) is presently undergoing a phase I trial combined with DEBTACE. The aim of this study is to look for the safest combination therapy at various stages. Up to 1 year, all participants will be treated with intravenous nivolumab (240 mg) every two weeks. TACE along with nivolumab is also studied in a phase II staged single-arm trial; it is an open-label study for intermediate-staged HCC (NCT 03572582), which concentrates on the degree of response. Initially TACE treatment will be distributed into cycles of 4 weeks. Day 1 (4 days) of cycle 3 (8 weeks and 4 days later), the following TACE will be repeated. Locoregional therapy can stimulate the preparation of expression of Th17 and T cells in the peripheral circulation by provoking the immunostimulating agents (IFN- $\gamma$, TNF- $\alpha$ ), simultaneously inhibiting immunosuppressive factors (IL-6). Irradiation treatment shrinks vessel density and expands cytotoxic $T$ lymphocyte permeability in the vascular system. It also increases the expression of VCAM-1 and promotes cytotoxic T cell infiltration, which boosts the anti-tumor immune response. There are currently numerous clinical trials ongoing or completed that use TACE and radio-frequency ablation combined with immunotherapy for hepatocellular carcinoma patients (Table I).

\section{Futuristic approach using combinational regimen for $\mathrm{HCC}$}

Apart from using ICB and RT in HCC therapy, the phase III staged trial of IMbrave150 in HCC

Table I. Selected ongoing clinical trials of use of TACE and radio-frequency ablation combined with immunotherapy for hepatocellular carcinoma patients (https://www.clinicaltrials.gov/)

\begin{tabular}{|lcc|}
\hline Therapies & Immunotherapeutic drugs or agents & Clinical trials \\
\hline TACE & Pembrolizumab & NCT03397654 \\
\hline TACE & Immuncell-LC & NCT02856815 \\
\hline TACE & Durvalumab and bevacizumab & NCT03937830 \\
\hline TACE & Immune checkpoint inhibitor & NCT03817736 \\
\hline TACE & Nivolumab or pembrolizumab & NCT03259867 \\
\hline TACE & DC-CIK & NCT02487017 \\
\hline TACE & CTAL-4/PD-L1 (durvalumab and & NCT03638141 \\
& tremelimumab) & \\
\hline TACE & Autologous Tcm immunotherapy & NCT03575806 \\
\hline TACE & Nivolumab & NCT03572582 \\
\hline TACE & Immune killer cells & NCT03592706 \\
\hline TACE & Cytokine-induced killer (CIK) & NCT02568748 \\
\hline Chemoembolization (TACE) or Ablation (RFA) & Tremelimumab & NCT01853618 \\
\hline Y90-Radioembolization & Nivolumab & NCT03033446 \\
\hline Ablation & Nivolumab & NCT03383458 \\
\hline Y90-Radioembolization & Pembrolizumab & NCT03099564 \\
\hline
\end{tabular}


patients showed superiority in OS, PFS and ORR when treated with anti-VEGF-A bevacizumab and anti-PD-L1 atezolizumab as compared to patients who underwent sorafenib treatment [84]. Angiogenesis is the major hallmark of cancer, a crucial component in tumor development and progression, and has been a promising target for drug evaluation in $\mathrm{HCC}[35,85,86]$. The vascular abnormalities seen in HCC tumors have been linked to dysregulated VEGF expression [86, 87]. The outcomes of other research experiments in HCC patients have demonstrated that increased levels of VEGF after RFA or surgery are directly linked to a weak prognosis $[88,89]$. Shigeta et al. explained the underlying pathway of the combinational treatment of anti-VEGF-A and anti-PD-L1 against tumor genesis using an orthotopic murine HCC model. They found that inhibiting VEGFR2- alone shows increased PD-1 levels on CD4+ cells in the tumor; however, when given in combination with anti-PD-1 therapy, the functioning of CD4+ cells was restored, leading to normal vessel formation. The combined therapy also enhances cytotoxic $\mathrm{CD} 8+\mathrm{T}$ cell activation and tumor infiltration while Treg and monocyte infiltration decreases [90].

The expression level of VEGF in RT treated HCC tumors was significantly elevated [89]. Depending on this, it is worthwhile to investigate the potency of treating HCC patients with ICB and anti-angiogenesis after RT because of their capability to strengthen tumor-killing activities and control the tumor cells to renew (Figure 4). A second strategy is using an anti-angiogenic drug before RT to "normalize" the tumor vasculature and, as a result, increase the efficacy of the radiotherapy effects [91]. Following this combination treatment, ICB could be given to maintain anti-tumor immunity (Figure 4). Anti-angiogenics are linked to serious

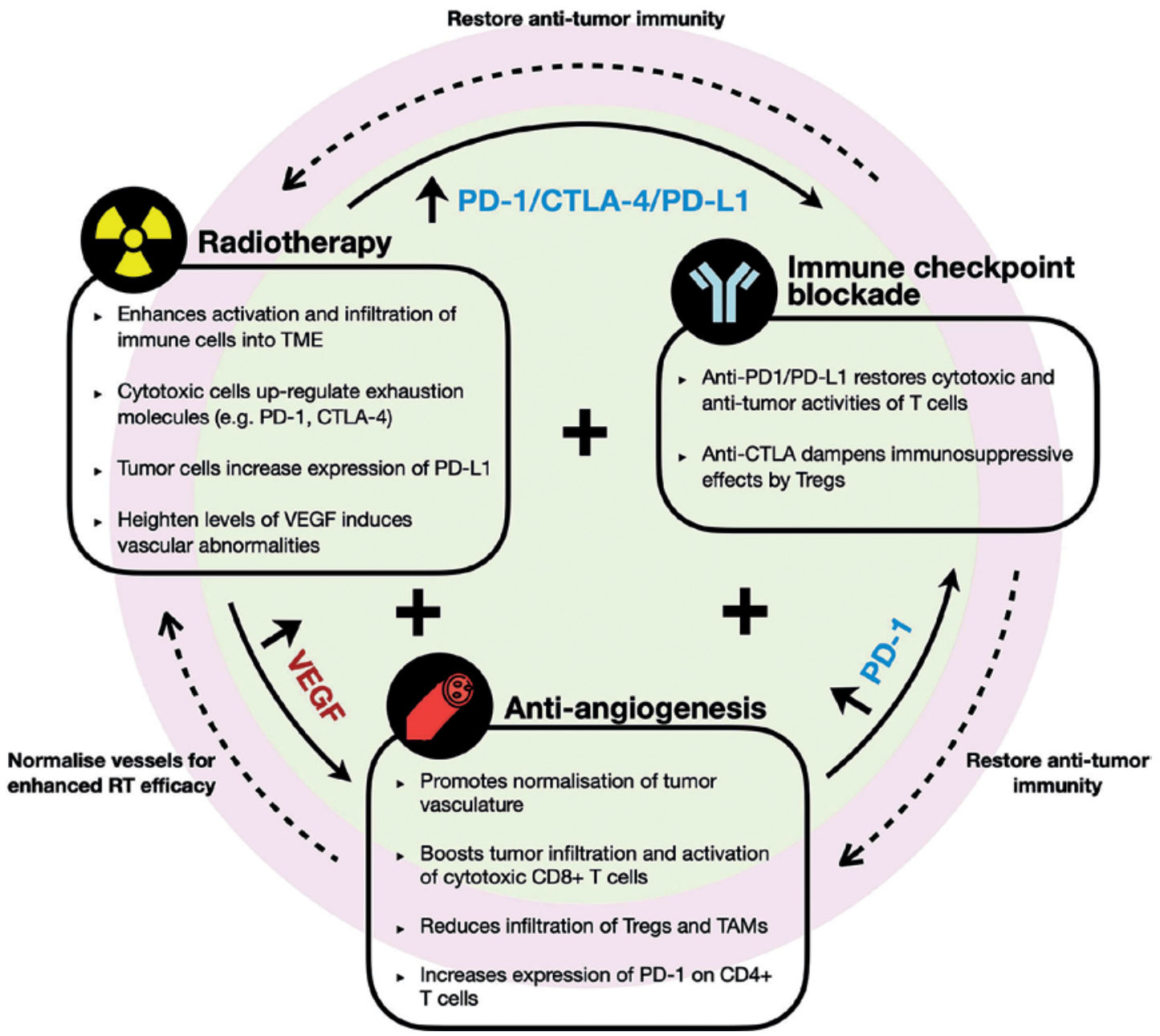

Figure 4. The hypothesis of combining immune checkpoint blockade, anti-angiogenesis agents and radiotherapy in a single treatment. The key role of immunomodulation of all the therapeutic agents, as well as the synergetic effect of their combination in HCC, is depicted in the diagram. Immune infiltrates into TME are increased by RT, and amplification of immune checkpoint agents (e.g., CTLA-4, PD-L1 and PD-1) and VEGF is shown. Anti-VEGF accelerates normal vessel formation, which raises RT potency and/or increases cytotoxic cell infiltration into TME and improves CD4+ T cell PD-1 levels. The synergistic role of ICB and RT improves the effect of anti-VEGF and RT therapies by restoring and further enhancing anti-tumor immune responses. Reproduced with permission from [39] 
gastrointestinal toxicity as a result of enhanced exposure to RT; therefore, careful evaluation of such treatment is required [92]. Other acceptable combinations such as dual RT with ICBs can be looked at as well.

\section{Conclusion and future perspective}

Evidence from the studies indicates that LRTs have better immune modulation properties in HCC patients, which strongly suggests that the potential and synergistic effect of combined treatment with immune checkpoint blockers should be investigated in depth. However, many unanswered questions arise regarding LRTs: when is the best time to start immunotherapy - before, during, or after? What biomarkers are the most effective at predicting the response to this combination? Is there a connection between PD-L1 status and response? Can immunotherapy induce substantial immune activation in early/locally advanced HCC to produce similar results? Any of these questions can be answered by ongoing research and multidisciplinary collaboration.

\section{Acknowledgments}

This work was supported by the Program from the Health and Family Planning Commission of Zhejiang Province (No. 2014KYA217 to RMX, No. $2019 \mathrm{KY} 727$ to ZQY) and the Program of Science and Technology (Medical and Health) from Shaoxing (No. 2018C30032 to LW).

Liang Wang and Zhong-Qiang Yu contributed equally to this work.

\section{Conflict of interest}

The authors declare no conflict of interest.

\section{References}

1. Ferenci P, Fried M, Labrecque D, et al. Hepatocellular carcinoma (HCC): a global perspective. J Clin Gastroenterol 2010; 44: 239-45.

2. Akinyemiju T, Abera S, Ahmed M, et al, The burden of primary liver cancer and underlying etiologies from 1990 to 2015 at the global, regional, and national level: results from the global burden of disease study 2015. JAMA Oncology 2017; 3: 1683-91.

3. Medavaram S, Zhang Y. Emerging therapies in advanced hepatocellular carcinoma. Exp Hematol Oncol 2018; 7: 17.

4. Bruix J, Qin S, Merle P, et al. Regorafenib for patients with hepatocellular carcinoma who progressed on sorafenib treatment (RESORCE): a randomised, double-blind, placebo-controlled, phase 3 trial. Lancet 2017; 389: 56-66.

5. Zhu AX, Kang YK, Yen CJ, et al. Ramucirumab after sorafenib in patients with advanced hepatocellular carcinoma and increased $\alpha$-fetoprotein concentrations (REACH-2): a randomised, double-blind, placebo-controlled, phase 3 trial. Lancet Oncol 2019; 20: 282-96.
6. Abou-Alfa GK, Meyer T, Cheng AL, et al. Cabozantinib in patients with advanced and progressing hepatocellular carcinoma. N Engl J Med 2018; 379: 54-63.

7. Food, U.S. and A. Drug, FDA grants accelerated approval to pembrolizumab for hepatocellular carcinoma. 2018.

8. Food, U.S, Drug Administration: FDA Grants Accelerated Approval to Nivolumab for HCC Previously Treated with Sorafenib. FDA. gov.

9. Medzhitov R. Origin and physiological roles of inflammation. Nature 2008; 454: 428-35.

10. Landskron G, De la Fuente M, Thuwajit P, Thuwajit C, Hermoso MA. Chronic inflammation and cytokines in the tumor microenvironment. J Immunol Res 2014; 2014: 149185.

11. Hanahan D, Weinberg RA. Hallmarks of cancer: the next generation. Cell 2011; 144: 646-74.

12. Lim CJ, Lee YH, Pan L, et al. Multidimensional analyses reveal distinct immune microenvironment in hepatitis B virus-related hepatocellular carcinoma. Gut 2019; 68: 916-27.

13. Zhu AX, Duda DG, Sahani DV, Jain RK. HCC and angiogenesis: possible targets and future directions. Nature Rev Clin Oncol 2011; 8: 292-301.

14. Balkwill F, Mantovani A. Inflammation and cancer: back to Virchow? Lancet 2001; 357: 539-45.

15. Das UN. Molecular Biochemical Aspects of Cancer. Springer 2020.

16. Noy R, Pollard JW. Tumor-associated macrophages: from mechanisms to therapy. Immunity 2014; 41: 49-61.

17. Mantovani A, Sozzani S, Locati M, Allavena P, Sica A. Macrophage polarization: tumor-associated macrophages as a paradigm for polarized M2 mononuclear phagocytes. Trends Immunol 2002; 23: 549-55.

18. Chew V, Lai L, Pan L, et al. Delineation of an immunosuppressive gradient in hepatocellular carcinoma using high-dimensional proteomic and transcriptomic analyses. Proc Natl Acad Sci USA 2017; 114: E5900-9.

19. Fu J, Xu D, Liu Z, et al. Increased regulatory T cells correlate with CD8 T-cell impairment and poor survival in hepatocellular carcinoma patients. Gastroenterology 2007; 132: 2328-39.

20. Farhood B, Najafi M, Mortezaee K. CD8+ cytotoxic T lymphocytes in cancer immunotherapy: a review. J Cell Physiol 2019; 234: 8509-21.

21. Zheng C, Zheng L, Yoo JK, et al. Landscape of infiltrating $T$ cells in liver cancer revealed by single-cell sequencing. Cell 2017; 169: 1342-56.

22. Gao Q, Qiu SJ, Fan J, et al. Intratumoral balance of regulatory and cytotoxic $\mathrm{T}$ cells is associated with prognosis of hepatocellular carcinoma after resection. J Clin Oncol 2007; 25: 2586-93.

23. Chen J, Gingold JA, Su X. Immunomodulatory TGF- $\beta$ signaling in hepatocellular carcinoma. Trends Mol Med 2019; 25: 1010-23.

24. Zhang JP, Yan J, Xu J, et al. Increased intratumoral IL-17-producing cells correlate with poor survival in hepatocellular carcinoma patients. J Hepatol 2009; 50: 980-9.

25. Quezada SA, Simpson TR, Peggs KS, et al. Tumor-reactive CD4+ T cells develop cytotoxic activity and eradicate large established melanoma after transfer into lymphopenic hosts. J Exp Med 2010; 207: 637-50.

26. Palucka K, Banchereau J. Cancer immunotherapy via dendritic cells. Nat Rev Cancer 2012; 12: 265-77.

27. LaCasse CJ, Janikashvili N, Larmonier CB, et al. Th-1 lymphocytes induce dendritic cell tumor killing activity by an IFN- $\gamma$-dependent mechanism. J Immunol 2011; 187: 6310-7. 
28. Lee HL, Jang JW, Lee SW, et al. Inflammatory cytokines and change of Th1/Th2 balance as prognostic indicators for hepatocellular carcinoma in patients treated with transarterial chemoembolization. Sci Rep 2019; 9: 3260

29. Foerster F, Hess M, Gerhold-Ay A, et al. The immune contexture of hepatocellular carcinoma predicts clinical outcome. Sci Rep 2018; 8: 1-11.

30. Ruffell B, DeNardo DG, Affara NI, Coussens LM. Lymphocytes in cancer development: polarization towards pro-tumor immunity. Cytokine Growth Factor Rev 2010; 21: 3-10.

31. DeNardo DG, Barreto JB, Andreu P, et al. CD4+ T cells regulate pulmonary metastasis of mammary carcinomas by enhancing protumor properties of macrophages. Cancer Cell 2009; 16: 91-102.

32. Saxena R, Kaur J. Th1/Th2 cytokines and their genotypes as predictors of hepatitis $B$ virus related hepatocellular carcinoma. World J Hepatol 2015; 7: 1572-80.

33. Das UN. A novel embolization technique to stem hemorrhage complications and cancer. Balkan Med J 2020; 37: 182-3.

34. Cai L, Zhang Z, Zhou L, et al. Functional impairment in circulating and intrahepatic NK cells and relative mechanism in hepatocellular carcinoma patients. Clin Immunol 2008; 129: 428-37.

35. Lim CJ, Lee YH, Pan L, et al. Multidimensional analyses reveal distinct immune microenvironment in hepatitis B virus-related hepatocellular carcinoma. Gut 2019; 68 : 916-27.

36. Chew V, Lai L, Pan L, et al. Delineation of an immunosuppressive gradient in hepatocellular carcinoma using high-dimensional proteomic and transcriptomic analyses. Proc Natl Acad Sci USA 2017; 114: E5900-9.

37. Barber DL, Wherry EJ, Masopust D, et al. Restoring function in exhausted CD8 T cells during chronic viral infection. Nature 2006; 439: 682-7.

38. Farhood B, Najafi M, Mortezaee K. CD8(+) cytotoxic T lymphocytes in cancer immunotherapy: a review. J Cell Physiol 2019; 234: 8509-21.

39. Lee YH, Tai D, Yip C, Choo SP, Chew V. Combinational immunotherapy for hepatocellular carcinoma: radiotherapy, immune checkpoint blockade and beyond. Front Immunol 2020; 11: 568759-9.

40. Zhu AX, Finn RS, Edeline J, et al. Pembrolizumab in patients with advanced hepatocellular carcinoma previously treated with sorafenib (KEYNOTE-224): a non-randomised, open-label phase 2 trial. Lancet Oncol 2018; 19: 940-52.

41. El-Khoueiry AB, Sangro B, Yau T, et al. Nivolumab in patients with advanced hepatocellular carcinoma (CheckMate 040): an open-label, non-comparative, phase $1 / 2$ dose escalation and expansion trial. Lancet 2017; 389: 2492-502.

42. Finn RS, Ryoo BY, Merle P, et al. Results of KEYNOTE-240: phase 3 study of pembrolizumab (Pembro) vs best supportive care (BSC) for second line therapy in advanced hepatocellular carcinoma (HCC). J Clin Oncol 2019; 37 (15 Suppl): 4004-4.

43. An Investigational Immuno-therapy Study of Nivolumab Compared to Sorafenib as a First Treatment in Patients With Advanced Hepatocellular Carcinoma. 1-03-2021; Available from: https://www.smartpatients.com/trials/ NCT02576509.

44. Wainberg ZA, Segal NH, Jaeger D, et al. Safety and clinical activity of durvalumab monotherapy in patients with hepatocellular carcinoma (HCC). J Clin Oncol 2017; 35 (15 Suppl): 4071.
45. Sangro B, Gomez-Martin C, de la Mata M, et al. A clinical trial of CTLA-4 blockade with tremelimumab in patients with hepatocellular carcinoma and chronic hepatitis C. J Hepatol 2013; 59: 81-8.

46. Abou-Alfa GK, Chan SL, Furuse J, et al. A randomized, multicenter phase 3 study of durvalumab (D) and tremelimumab $(T)$ as first-line treatment in patients with unresectable hepatocellular carcinoma (HCC): HIMALAYA study. J Clin Oncol 2018; 36 (15 Suppl): TPS4144.

47. Finn RS, Ducreux M, Qin S, et al. IMbrave150: a randomized phase III study of $1 \mathrm{~L}$ atezolizumab plus bevacizum$a b$ vs sorafenib in locally advanced or metastatic hepatocellular carcinoma. J Clin Oncol 2018; 36 (15 Suppl): TPS4141.

48. Chu KF, Dupuy DE. Thermal ablation of tumours: biological mechanisms and advances in therapy. Nat Rev Cancer 2014; 14: 199-208.

49. den Brok MH, Sutmuller Rp, van der Voort R, et al. In situ tumor ablation creates an antigen source for the generation of antitumor immunity. Cancer Res 2004; 64: 4024-9.

50. Jansen MC, van Hillegersberg R, Schoots IG, et al. Cryoablation induces greater inflammatory and coagulative responses than radiofrequency ablation or laser induced thermotherapy in a rat liver model. Surgery 2010; 147: 686-95.

51. Edd JF, Horowitz L, Davalos RV, Mir LM, Rubinsky B. In vivo results of a new focal tissue ablation technique: irreversible electroporation. IEEE Trans Biomed Eng 2006; 53: 1409-15.

52. Sugimoto K, Kakimi K, Takeuchi $\mathrm{H}$, et al. Irreversible electroporation versus radiofrequency ablation: comparison of systemic immune responses in patients with hepatocellular carcinoma. J Vasc Interv Radiol 2019; 30: 845-53.

53. Keisari Y. Tumor abolition and antitumor immunostimulation by physico-chemical tumor ablation. Front Biosci 2017; 22: 310-47.

54. Haen SP, Gouttefangeas C, Schmidt D, et al. Elevated serum levels of heat shock protein 70 can be detected after radiofrequency ablation. Cell Stress Chaperones 2011; 16: 495-504.

55. Thimme R, Neagu M, Boettler T, et al. Comprehensive analysis of the alpha-fetoprotein-specific CD8+ T cell responses in patients with hepatocellular carcinoma. Hepatology 2008; 48: 1821-33.

56. Narasimha Das U. Lipoxins, resolvins, protectins, maresins and nitrolipids, and their clinical implications with specific reference to cancer: part I. Clin Lipidol 2013; 8: 437-63.

57. Rozenblum N, Zeira E, Bulvik B, et al. Radiofrequency ablation: inflammatory changes in the periablative zone can induce global organ effects, including liver regeneration. Radiology 2015; 276: 416-25.

58. Li X, Liang P. Immunotherapy for hepatocellular carcinoma following thermal ablation. J Buon 2014; 19: 867-71.

59. Gravante G, Sconocchia G, Ong SL, Dennison AR, Lloyd DM. Immunoregulatory effects of liver ablation therapies for the treatment of primary and metastatic liver malignancies. Liver Int 2009; 29: 18-24.

60. Dong BW, Zhang J, Liang P, et al. Sequential pathological and immunologic analysis of percutaneous microwave coagulation therapy of hepatocellular carcinoma. Int J Hyperthermia 2003; 19: 119-33.

61. Chen MF, Chen PT, Chen WC, et al. The role of PD-L1 in the radiation response and prognosis for esophageal squamous cell carcinoma related to IL-6 and T-cell immunosuppression. Oncotarget 2016; 7: 7913-24. 
62. Duan XH, Li H, Han XW, et al. Upregulation of IL-6 is involved in moderate hyperthermia induced proliferation and invasion of hepatocellular carcinoma cells. Eur J Pharmacol 2018; 833: 230-6.

63. Sharabi AB, Lim M, DeWeese T, Drake CG. Radiation and checkpoint blockade immunotherapy: radiosensitisation and potential mechanisms of synergy. Lancet Oncol 2015; 16: e498-509.

64. Deng L, Liang H, Burnette B, et al. Irradiation and an ti-PD-L1 treatment synergistically promote antitumor immunity in mice. J Clin Invest 2014; 124: 687-95.

65. Park SS, Dong H, Liu X, et al. PD-1 Restrains radiotherapy-induced abscopal effect. Cancer Immunol Res 2015; 3: 610-9.

66. Zeng J, Harris TJ, Lim M, et al. Immune modulation and stereotactic radiation: improving local and abscopal responses. Biomed Res Int 2013; 2013: 658126.

67. Kim KJ, Kim JH, Lee SJ, et al. Radiation improves antitumor effect of immune checkpoint inhibitor in murine hepatocellular carcinoma model. Oncotarget 2017; 8 : 41242-55.

68. Son CH, Bae JH, Shin DY, et al. Combination effect of regulatory T-cell depletion and ionizing radiation in mouse models of lung and colon cancer. Int J Radiat Oncol Biol Phys 2015; 92: 390-8.

69. Rasool M, Rashid S, Arooj M, et al. New possibilities in hepatocellular carcinoma treatment. Anticancer Res 2014; 34: 1563-71.

70. Bujold A, Dawson LA. Stereotactic radiation therapy and selective internal radiation therapy for hepatocellular carcinoma. Cancer Radiother 2011; 15: 54-63.

71. Chew V, Lee YH, Pan L, et al. Immune activation underlies a sustained clinical response to Yttrium-90 radioembolisation in hepatocellular carcinoma. Gut 2019; 68 : 335-46.

72. Zeng P, Shen D, Zeng CH, Chang XF, Teng GJ. Emerging opportunities for combining locoregional therapy with immune checkpoint inhibitors in hepatocellular carcinoma. Curr Oncol Rep 2020; 22: 76

73. Ayaru L, Pereira SP, Alisa A, et al. Unmasking of alpha-fetoprotein-specific CD4(+) T cell responses in hepatocellular carcinoma patients undergoing embolization. J Immunol 2007; 178: 1914-22.

74. Das UN. Occlusion of infusion vessels on $\gamma$-linolenic acid infusion. Prostaglandins Leukotrienes Essential Fatty Acids 2004; 70: 23-32.

75. Lee HL, Jang JW, Lee SW, et al. Inflammatory cytokines and change of Th1/Th2 balance as prognostic indicators for hepatocellular carcinoma in patients treated with transarterial chemoembolization. Sci Rep 2019; 9: 3260.

76. Liao J, Xiao J, Zhou Y, Liu Z, Wang C. Effect of transcatheter arterial chemoembolization on cellular immune function and regulatory $T$ cells in patients with hepatocellular carcinoma. Mol Med Rep 2015; 12: 6065-71.

77. Zhang J, Li H, Gao D, et al. A prognosis and impact factor analysis of DC-CIK cell therapy for patients with hepatocellular carcinoma undergoing postoperative TACE. Cancer Biol Ther 2018; 19: 475-83.

78. Das UN. Bioactive lipids as modulators of immune check point inhibitors. Med Hypotheses 2020; 135: 109473.

79. Miyayama S. Treatment strategy of transarterial chemoembolization for hepatocellular carcinoma. Appl Sci 2020; 10: 7337.

80. Semaan A, Dietrich D, Bergheim D, et al. CXCL12 expression and PD-L1 expression serve as prognostic biomark ers in HCC and are induced by hypoxia. Virchows Arch 2017; 470: 185-96.
81. Duffy AG, Ulahannan SV, Makorova-Rusher O, et al. A pilot study of tremelimumab, a monoclonal antibody against CTLA-4, in combination with either transcatheter arterial chemoembolization (TACE) or radiofrequency ablation (RFA) in patients with hepatocellular carcinoma (HCC). J Clin Oncol 2014; 32 (15 Suppl): e15133.

82. Greten TF, Mauda-Havakuk M, Heinrich B, Korangy F, Wood BJ. Combined locoregional-immunotherapy for liver cancer. J Hepatol 2019; 70: 999-1007.

83. Zhang T, Chen J, Niu L, et al. Clinical safety and efficacy of locoregional therapy combined with adoptive transfer of allogeneic $\gamma \delta$ T cells for advanced hepatocellular carcinoma and intrahepatic cholangiocarcinoma. J Vasc Interv Radiol 2021.

84. Cheng AL, Qin S, Ikeda M, et al. LBA3 - IMbrave150: Efficacy and safety results from a ph III study evaluating atezolizumab (atezo) + bevacizumab (bev) vs sorafenib (Sor) as first treatment (tx) for patients (pts) with unresectable hepatocellular carcinoma (HCC). Ann Oncol 2019; 30: ix186-7.

85. Hanahan D, Weinberg RA. Hallmarks of cancer: the next generation. Cell 2011; 144: 646-74.

86. Zhu AX, Duda DG, Sahani DV, Jain RK. HCC and angiogenesis: possible targets and future directions. Nat Rev Clin Oncol 2011; 8: 292-301.

87. LeCouter J, Moritz DR, Li B, et al. Angiogenesis-independent endothelial protection of liver: role of VEGFR-1. Science 2003; 299: 890-3.

88. Chao Y, Li CP, Chau GY, et al. Prognostic significance of vascular endothelial growth factor, basic fibroblast growth factor, and angiogenin in patients with resectable hepatocellular carcinoma after surgery. Ann Surg Oncol 2003; 10: 355-62.

89. Suh YG, Lee EJ, Cha H, Yang SH, Seong J. Prognostic values of vascular endothelial growth factor and matrix metalloproteinase-2 in hepatocellular carcinoma after radiotherapy. Dig Dis 2014; 32: 725-32.

90. Shigeta K, Datta M, Hato T, et al. Dual programmed death receptor- 1 and vascular endothelial growth factor receptor-2 blockade promotes vascular normalization and enhances antitumor immune responses in hepatocellular carcinoma. Hepatology 2020; 71: 1247-61.

91. Wu JB, Tang YL, Liang XH. Targeting VEGF pathway to normalize the vasculature: an emerging insight in cancer therapy. Onco Targets Ther 2018; 11: 6901-9.

92. Pollom EL, Deng L, Pai RK, et al. Gastrointestinal toxicities with combined antiangiogenic and stereotactic body radiation therapy. Int J Radiat Oncol Biol Phys 2015; 92: 568-76. 